\title{
Characterization of sputum biomarkers for asthma-COPD overlap syndrome
}

\author{
This article was published in the following Dove Press journal: \\ International Journal of COPD \\ 30 September 2016 \\ Number of times this article has been viewed
}

\section{Jing Gao' \\ Hiroshi Iwamoto² \\ Jukka Koskela ${ }^{3}$ \\ Harri Alenius ${ }^{4}$ \\ Noboru Hattori ${ }^{2}$ \\ Nobuoki Kohno ${ }^{5}$ \\ Tarja Laitinen ${ }^{6}$ \\ Witold Mazur' \\ Ville Pulkkinen'}

'Heart and Lung Center, University of Helsinki and Helsinki University

Hospital, Helsinki, Finland;

${ }^{2}$ Department of Molecular and Internal Medicine, Graduate School of Biomedical Sciences, Hiroshima University, Hiroshima, Japan; ${ }^{3}$ Clinical Research Unit of Pulmonary Diseases and Division of Pulmonology, Heart and Lung Center, University of Helsinki and Helsinki University Hospital, ${ }^{4}$ Unit of Systems Toxicology, Finnish Institute of Occupational Health, Helsinki, Finland; ${ }^{5}$ Hiroshima Cosmopolitan University, Hiroshima, Japan; ${ }^{6}$ Department of Pulmonary Diseases and Clinical Allergology, Turku University Hospital, University of Turku, Turku, Finland

Correspondence: Ville Pulkkinen University of Helsinki, Biomedicum, PO Box 63, 00014, Helsinki, Finland Tel +358 504270555

Email ville.pulkkinen@helsinki.fi

\begin{abstract}
Asthma-COPD overlap syndrome (ACOS) is a commonly encountered chronic airway disease. However, ACOS is still a consensus-based clinical phenotype and the underlying inflammatory mechanisms are inadequately characterized. To clarify the inflammatory mediatypical for ACOS, five biomarkers, namely interleukin (IL)-13, myeloperoxidase (MPO), neutrophil gelatinase-associated lipocalin (NGAL), chitinase-like protein (YKL-40), and IL-6, were selected. This study hypothesized that sputum biomarkers relevant for airway inflammation in asthma (IL-13), COPD (MPO, NGAL), or in both asthma and COPD (YKL-40, IL-6) could be used to differentiate ACOS from COPD and asthma. The aim of this study was to characterize the inflammatory profile and improve the recognition of ACOS. Induced sputum levels of IL-13, MPO, NGAL, YKL-40, and IL-6 were measured by enzyme-linked immunosorbent assay/Luminex assay in a Finnish discovery cohort $(n=90)$ of nonsmokers, smokers, and patients with asthma, COPD, and ACOS and validated in a Japanese cohort $(n=135)$. The classification accuracy of potential biomarkers was compared with area under the receiver operating characteristic curves. Only sputum NGAL levels could differentiate ACOS from asthma $(P<0.001$ and $P<0.001)$ and COPD $(P<0.05$ and $P=0.002)$ in the discovery and replication cohorts, respectively. Sputum NGAL levels were independently correlated with the percentage of prebronchodilator forced expiratory volume in 1 second predicted in multivariate analysis in the discovery and replication cohorts $(P=0.001$ and $P=0.002$, respectively). In conclusion, sputum biomarkers reflecting both airway inflammation and remodeling of the tissue show potential in differentiation between asthma, COPD, and ACOS.
\end{abstract}

Keywords: COPD pathology, asthma, ACOS

\section{Introduction}

The asthma-COPD overlap syndrome (ACOS) has recently been recognized as a consensus-based clinical phenotype sharing the features of asthma and COPD. ${ }^{1-3}$ Approximately $20 \%-50 \%$ of patients with irreversible airway obstruction show airflow variability characteristic to asthma. ${ }^{4-7}$ Differential diagnosis of ACOS from asthma and COPD is increasingly important, since ACOS has a poor prognosis and different treatment guidelines. ACOS is associated with low health-related quality of life, ${ }^{6}$ increased exacerbation rate and hospital admissions, a rapid decline in lung function, and high mortality. ${ }^{5,6,8}$ However, ACOS cannot be diagnosed unambiguously on the basis of lung function tests, patients' demographics, and sputum cell counts or by imaging of the lungs. ${ }^{6,9,10}$ There is an unmet need to develop novel diagnostic biomarkers for clinical assessment of ACOS.

Although the inflammatory pathways of asthma and COPD are markedly different, ${ }^{11,12}$ they coincide in ACOS, and both eosinophils and neutrophils in sputum are characteristic for ACOS. Clinically, the main interest would be to identify those 
patients with ACOS who could be responsive to inhaled corticosteroids. ${ }^{13}$ For this, the assessment of fractional exhaled nitric oxide or immunoglobulin $\mathrm{E}$ in COPD patients could be useful. ${ }^{14}$ For the current study, interleukin (IL)-13, ${ }^{15,16}$ which is a T-helper 2 type marker known to be important in eosinophilic inflammation and asthma, was analyzed. In addition, chitinase-like protein (YKL-40), ${ }^{17,18}$ which is elevated in both asthma and COPD, was selected. ${ }^{19}$ IL-6 was selected because its serum and sputum levels are associated with impaired lung function, ${ }^{15,20,21}$ and elevated serum IL-6 levels were detected in patients with ACOS. ${ }^{22}$ In addition, myeloperoxidase (MPO) and neutrophil gelatinase-associated lipocalin (NGAL) were assessed based on the authors' earlier findings that suggested enhanced neutrophil-mediated inflammation and/or airway epithelial injury in ACOS. ${ }^{9}$

This study hypothesized that sputum biomarkers relevant for airway inflammation in asthma (IL-13), COPD (MPO, NGAL), or in both asthma and COPD (YKL-40, IL-6) could be used to differentiate ACOS from COPD and asthma and further could be used to improve the determination and recognition of ACOS.

\section{Subjects and methods Subjects}

The discovery cohort consisted of 90 volunteer individuals who were a part of the cohort previously reported in Iwamoto et al. ${ }^{9}$ The cohort size was smaller than the original one because of the consumption of sputum samples. The patients, who were a part of the longitudinally followed cohort of Finnish asthma and COPD patients (FinnCADStudy), 6,9,23 were recruited from the Helsinki University Hospital. Based on their medical history and self-reported questionnaire data, the subjects were categorized into five groups: healthy nonsmokers (NSs, $n=14$ ), healthy smokers (HSs, $n=14$ ), and patients with asthma $(n=24), \operatorname{COPD}(n=20)$, and ACOS $(n=18)$. Spirometry with a bronchodilator test and the diffusing capacity of the lung for carbon monoxide were performed on all participants. The control subjects (NSs and HSs) consisted of responders to an advertisement in the Helsinki University Hospital and the local media. These participants were asymptomatic, never, or current smokers with no history of lung disease confirmed by a normal lung function with post-bronchodilator forced expiratory volume in 1 second $\left(\mathrm{FEV}_{1}\right) /$ forced vital capacity $(\mathrm{FVC}) \geq 0.7$ in spirometry.

The diagnosis of asthma was based on the British Guidelines on Asthma Management ${ }^{24}$ and defined as reversible airflow obstruction with a post-bronchodilator $\mathrm{FEV}_{1}$ / FVC $\geq 0.7$, with one or more of the following findings: a post-bronchodilator increase in $\mathrm{FEV}_{1}$ of $\geq 12 \%$, a bronchodilator response of $\geq 15 \%$ or diurnal variation of $\geq 20 \%$ in peak expiratory flow recording, moderate-to-severe bronchial hyperreactivity, or a decrease in $\mathrm{FEV}_{1}$ of $\geq 15 \%$ in the exercise test. COPD was diagnosed according to the American Thoracic Society/European Respiratory Society Task Force recommendations ${ }^{23,25,26}$ and defined by incompletely reversible airflow obstruction with a post-bronchodilator $\mathrm{FEV}_{1}$ / FVC $<0.7 .{ }^{1}$ ACOS was identified by the features that it shares with both asthma and COPD according to the Global Initiative for Asthma guidelines ${ }^{2}$ and Global Initiative for Obstructive Lung Diseases strategy. ${ }^{1}$ In the ACOS group, the age of onset was $>40$ years and all the patients had history of smoking. The patients with ACOS had increased airflow variability and incompletely reversible airflow obstruction. Patients with COPD and ACOS had no history of alpha-1antitrypsin deficiency in their family. All patients had respiratory symptoms and were current or former smokers ( $>10$ pack-years) when developed their lung disease. None of the patients received oral glucocorticoid treatment or experienced an exacerbation during the study period. Signs of atopy were not included in the diagnostic criteria.

The Japanese replication cohort $(n=135)$ consisted of NSs $(n=22)$, HSs $(n=40)$, and patients with asthma $(n=21)$, COPD $(n=35)$, and ACOS $(n=17)$. The diagnosis of asthma was assessed by specialists in respiratory medicine using the Global Initiative for Asthma guidelines. ${ }^{2}$ COPD diagnosis was based on long-term smoking ( $>10$ pack-years) and a prebronchodilator $\mathrm{FEV}_{1} / \mathrm{FVC}<0.7 .^{1,27,28}$ The individuals in the ACOS group consisted of patients who had been diagnosed with both asthma and COPD. All the patients with asthma, COPD, and ACOS were in stable condition and were using regular inhaled medications. For healthy control subjects, only prebronchodilator spirometries were performed, and their prebronchodilator $\mathrm{FEV}_{1} / \mathrm{FVC}$ values were $>0.7$.

This study was approved by the Ethics Committees of Helsinki University Central Hospital and Hiroshima University and conducted in accordance with the ethical standards established in the 1975 Declaration of Helsinki. All participants provided written informed consent.

\section{Sampling of sputum}

Sputum was induced by inhalation of hypertonic saline and treated with dithioerythritol (Sigma-Aldrich Co., St Louis, MO, USA) as recommended by the European Respiratory Society Task Force and described earlier in detail. 9,29,30 The supernatant was frozen at $-80^{\circ} \mathrm{C}$ for biochemical analyses. Cell viability was studied with Trypan blue in a Burker 
chamber. ${ }^{31}$ Cytocentrifuge preparations were made by Cytospin (Shandon Cytospin 3) and centrifuged at $450 \mathrm{rpm}$ for 6 minutes. The slides were stained with May-GrunwaldGiemsa solution (EMD Millipore, Billerica, MA, USA) for cell differential counts. Detailed cell profiles were assessed based on 400 cells counted from each slide. Only the representative samples with $<70 \%$ of squamous epithelial cells were accepted for the assessments. ${ }^{9,31}$ The slides were frozen at $-20^{\circ} \mathrm{C}$. Induction of the sputum samples in the Japanese cohort has been described earlier. ${ }^{32}$

\section{Enzyme-linked immunosorbent assay and Luminex assays}

YKL-40 was measured by Quantikine ELISA (R\&D Systems, Inc., Minneapolis, MN, USA), IL-6 by Magnetic Human High Sensitivity Luminex assay (R\&D Systems, Inc.), IL-13 by enzyme-linked immunosorbent assay (ELISA; USCN Life Science Inc., Wuhan, People's Republic of China), MPO by ELISA (Abnova Inc., Walnut, CA, USA), and NGAL by ELISA (USCN Life Science Inc.) according to the manufacturers' instructions. The minimal detectable dose for YKL-40, IL-6, IL-13, MPO, and NGAL were $3.55 \mathrm{pg} / \mathrm{mL}, 0.14 \mathrm{pg} / \mathrm{mL}, 5.7 \mathrm{pg} / \mathrm{mL}, 0.78 \mathrm{ng} / \mathrm{mL}$, and $17 \mathrm{pg} / \mathrm{mL}$, respectively. The analysis of MPO and NGAL in the discovery cohort has been performed earlier but added in the present analysis to identify the most informative panel of biomarkers. ${ }^{9}$

\section{Statistical analysis}

The demographics are expressed as mean \pm standard deviation, and the biomarker concentrations as medians with interquartile range. All statistical analyses were performed with the SPSS 20.0 software program (IBM Corporation, Armonk, NY, USA). Comparisons between groups were evaluated by the Kruskal-Wallis test followed by the MannWhitney $U$ test, when appropriate. Sputum biomarkers were further analyzed by the area under the curve (AUC) statistics for their predictive capability to distinguish ACOS from asthma, and ACOS and asthma from COPD or NSs. Spearman's correlations of the induced sputum marker levels with age, lung function, and sputum cell counts were calculated. Multivariate stepwise regression analysis was performed to obtain the variables to model ACOS. Due to the differences in sputum processing, such as a single versus three times inhalation of hypertonic saline in the discovery and replication cohort, respectively, the concentrations of sputum biomarkers were not directly comparable between the two cohorts. To make the comparison feasible, raw data was used in the comparisons within each cohort and standardized data ( $Z$-scores) in the comparisons between the two cohorts. $P$-values of $<0.05$ were considered statistically significant.

\section{Results \\ Subject characteristics}

The characteristics of the study subjects in the discovery cohort are shown in Table 1 . Healthy controls were $\sim 10$ years younger than the patients. Patients with COPD and ACOS had more pack-years and significantly lower FEV $\mathrm{FEV}_{1} / \mathrm{FVC}$ ratio, and diffusing capacity of the lung for carbon monoxide compared with those in the NS and asthma groups (Table 1). The patients with ACOS had significant reversibility in the bronchodilation test and diurnal variation of peak expiratory flow compared with those among the COPD patients. None of the participants had experienced an exacerbation or a respiratory tract infection within a month prior the study.

Successfully induced sputum samples were obtained from 90 individuals. The representativeness of samples was confirmed by cytospin centrifugation cell profiles. As expected, patients in the asthma $(n=24)$ and $\operatorname{ACOS}(n=18)$ groups had a higher percentage of sputum eosinophil compared with that in the HS group $(n=14)$. In addition, COPD $(n=20)$ and ACOS groups had a higher percentage of sputum neutrophils compared with that in the NS $(n=14)$ and HS groups (Table 1).

The characteristics of the study subjects in the replication cohort are shown in Table 2. There were no differences in pre-FEV $/$ /FVC, pack-years, neutrophil\%, and eosinophil\% between the patients with ACOS in the replication and in the discovery cohorts. However, patients with ACOS had greater pre-FEV $\%$ and pre-FVC\% values in the replication cohort than in the discovery cohort $(P=0.001$ and $P<0.001$, respectively).

\section{Sputum MPO and IL- I 3 were elevated in ACOS when compared with healthy controls}

The sputum levels of MPO and IL-13 were significantly increased in the ACOS groups when compared with the levels in the NS ( $P=0.012$ and $P<0.001$, respectively) and HS groups ( $P=0.008$ and $P<0.001$, respectively; Table 1$)$. There was also a significant difference in sputum MPO levels between the ACOS and asthma groups $(P=0.004)$. However, the sputum MPO and IL-13 levels could not distinguish ACOS from COPD $(P=0.128$ and $P=0.088$, respectively). 
Table I Clinical characteristics and biomarker concentrations of the study subjects across the discovery cohort $(n=90)$

\begin{tabular}{|c|c|c|c|c|c|c|}
\hline Discovery cohort & $\begin{array}{l}\text { Nonsmokers } \\
(n=14)\end{array}$ & $\begin{array}{l}\text { Healthy } \\
\text { smokers }(n=14)\end{array}$ & $\begin{array}{l}\text { Asthma } \\
(n=24)\end{array}$ & $\begin{array}{l}\text { COPD } \\
(n=20)\end{array}$ & $\begin{array}{l}A C O S \\
(n=18)\end{array}$ & $P$-value \\
\hline \multicolumn{7}{|l|}{ Demographics } \\
\hline Female/male & $5 / 9$ & $5 / 9$ & $15 / 9$ & $8 / 12$ & $6 / 12$ & \\
\hline Age (years) & $46.2(15.4)$ & $48.4(10.9)$ & $58.3(8.3)$ & $59.3(7.0)$ & $61.8(6.5)$ & $<0.001$ \\
\hline BMI $\left(k g / m^{2}\right)$ & $28.2(4.6)$ & $26.6(4.8)$ & $26.0(4.5)$ & $24.6(4.0)$ & $28.2(6.5)$ & 0.263 \\
\hline Allergy, n (\%) & I (7) & $7(50)$ & II (46) & $9(45)$ & $6(33)$ & \\
\hline ICS use, n (\%) & - & - & $20(83)$ & $9(45)$ & I3 (72) & \\
\hline \multicolumn{7}{|l|}{ Smoking status } \\
\hline Former/current & - & $0 / 14$ & $9 / 6$ & $7 / 12$ & $11 / 7$ & \\
\hline Pack-years & - & $27.1(16.1)$ & $13.0(16.0)$ & $37.2(17.3)$ & $42.5(17.7)$ & $<0.001$ \\
\hline \multicolumn{7}{|l|}{ Prebronchodilator } \\
\hline FVC (L) & $4.8(I . I)$ & $4.7(\mathrm{I} .4)$ & $3.1(0.7)$ & $3.3(1.0)$ & $3.0(1.1)$ & $<0.001$ \\
\hline FVC\% predicted & $103.6(9.4)$ & $98.6(15.1)$ & $81.1(17.7)$ & $81.7(16.5)$ & $69.3(18.6)$ & $<0.001$ \\
\hline $\mathrm{FEV}_{1}(\mathrm{~L})$ & $4.0(1.0)$ & $3.8(1.1)$ & $2.3(0.6)$ & $1.9(0.8)$ & $1.5(0.6)$ & $<0.001$ \\
\hline $\mathrm{FEV}_{1} \%$ predicted & I04.I (11.0) & $96.5(15.3)$ & $74.0(15.6)$ & $56.9(18.7)$ & $44.8(14.8)$ & $<0.001$ \\
\hline $\mathrm{FEV}_{1} / \mathrm{FVC} \%$ & $82.1(3.7)$ & $79.4(5.2)$ & $74.2(5.0)$ & $56.4(13.6)$ & $53.6(10.7)$ & $<0.001$ \\
\hline \multicolumn{7}{|l|}{ Post-bronchodilator } \\
\hline FVC (L) & $4.8(I . I)$ & $4.7(\mathrm{I} .4)$ & $3.2(0.7)$ & $3.4(0.9)$ & $3.3(1.0)$ & $<0.001$ \\
\hline FVC\% predicted & $102.6(8.9)$ & $98.8(15.9)$ & $83.1(14.7)$ & $83.4(16.7)$ & $75.5(16.9)$ & $<0.001$ \\
\hline $\mathrm{FEV}_{1}(\mathrm{~L})$ & $4.0(1.0)$ & $3.8(I . I)$ & $2.5(0.6)$ & $1.9(0.8)$ & $1.8(0.6)$ & $<0.001$ \\
\hline $\mathrm{FEV}_{1} \%$ predicted & $105.9(10.6)$ & $98.5(15.5)$ & $78.8(14.0)$ & $58.3(19.1)$ & $51.6(13.7)$ & $<0.001$ \\
\hline $\mathrm{FEV}_{1} / \mathrm{FVC} \%$ & $84.4(3.7)$ & $80.9(5.0)$ & $76.8(4.4)$ & $55.6(12.4)$ & $55.7(10.8)$ & $<0.001$ \\
\hline DLCO\% predicted & $99.0(9.0)$ & $89.8(10.2)$ & $82.2(17.8)$ & $53.2(20.2)$ & $67.9(18.7)$ & $<0.001$ \\
\hline \multicolumn{7}{|l|}{ Reversibility test } \\
\hline$\Delta \mathrm{FEV}_{1}^{\mathrm{a}}(\mathrm{mL})$ & $72.9(175.9)$ & $79.3(203.2)$ & I46.3 (166.8) & $53.5(80.6)$ & $231.1(187.4)$ & 0.004 \\
\hline$\Delta \mathrm{FEV}_{1} \% \mathrm{a}$ & $1.9(4.6)$ & $2.2(6.0)$ & $6.5(7.4)$ & $2.8(4.4)$ & $17.6(15.6)$ & $<0.001$ \\
\hline PEF diurnal variability (\%) & & & $25.6(12.1)$ & | $3.5(5.8)$ & $27.5(13.6)$ & 0.003 \\
\hline \multicolumn{7}{|c|}{ Differential sputum cell counts (\%) } \\
\hline Eosinophils & $1.9(3.8)$ & $0.3(0.6)$ & $5.1(9.2)$ & $2.4(4.9)$ & $6.8(9.8)$ & 0.001 \\
\hline Neutrophils & $36.8(8.0)$ & $42.5(20.8)$ & $53.6(23.4)$ & $62.8(20.3)$ & $64.8(15.5)$ & $<0.001$ \\
\hline \multicolumn{7}{|c|}{ Sputum biomarker concentrations } \\
\hline IL-6 (pg/mL) & I8.6 (8.1, 49.5) & $102.0(78.0,137.2)$ & $77.5(30.8,121.2)$ & $286.3(6|.6,4| 2.0)$ & $605.7(291.8,808.7)$ & $<0.001$ \\
\hline YKL-40 (ng/mL) & $22.5(8.9,44.0)$ & $15.0(8.7,35.0)$ & $47.8(22.1,100.5)$ & $50.4(37.7,64.4)$ & |42.4 (97.5, 52।.2) & $<0.001$ \\
\hline $\mathrm{IL}-\mathrm{I} 3(\mathrm{pg} / \mathrm{mL})$ & $53.9(27.6,82.4)$ & $40.7(29.4,50.1)$ & $159.0(57.7,332.4)$ & I4I.6 (86.6, 255.7) & $256.5(133.6,499.1)$ & $<0.001$ \\
\hline $\mathrm{NGAL}(\mu g / \mathrm{mL})$ & I.2 $(0.9,3.7)$ & $2.0(I .1,3.5)$ & $2.3(1.4,3.6)$ & $3.9(2.4,5.7)$ & $5.2(4.1,14.2)$ & $<0.001$ \\
\hline $\mathrm{MPO}(\mu \mathrm{g} / \mathrm{mL})$ & $2.9(2.1,16.8)$ & $4.3(1.5,14.0)$ & $6.3(3.8,11.7)$ & I2.I $(5.8,22.7)$ & $21.7(10.9,29.6)$ & 0.005 \\
\hline
\end{tabular}

Notes: The demographics are presented as mean (SD) unless otherwise stated. The biomarker concentrations are presented as medians (IQR). $P$-value for the differences was analyzed by Kruskal-Wallis $H$ test. $\Delta$, change in. ${ }^{a}$ Improvement in FEV, after $400 \mu g$ of salbutamol.

Abbreviations: ACOS, asthma-COPD overlap syndrome; BMI, body mass index; DLCO, diffusing capacity of the lung for carbon monoxide; FEV ${ }_{1}$, forced expiratory volume in I second; FVC, forced vital capacity; ICS, inhaled corticosteroids; IL, interleukin; IQR, interquartile range; MPO, myeloperoxidase; NGAL, neutrophil gelatinase-associated lipocalin; PEF, peak expiratory flow; SD, standard deviation; YKL-40, chitinase-3-like protein I.

\section{Sputum YKL-40, IL-6, and NGAL were elevated in ACOS when compared with COPD and asthma}

The sputum levels of YKL-40, IL-6, and NGAL were significantly increased in ACOS when compared with the NS $(P<0.001, P<0.001, P<0.001$, respectively) and HS groups $(P<0.001, P=0.001$, and $P=0.001$, respectively; Table 1). In addition, the sputum levels of YKL-40, IL-6, and NGAL were significantly increased in ACOS when compared with the asthma ( $P=0.001, P<0.001$, and $P<0.001$, respectively) and COPD groups ( $P=0.002, P=0.006$, and $P=0.026$, respectively). Sputum IL-6 and NGAL were also significantly increased in the COPD group when compared with the asthma group ( $P=0.034$ and $P=0.025$, respectively), while sputum YKL-40 levels were not significantly different between the COPD and asthma groups $(P>0.05)$.

\section{Sputum YKL-40, IL-6, and NGAL could differentiate ACOS from COPD} and asthma

A receiver operating characteristic curve analysis was carried out to evaluate the sensitivity, specificity, and diagnostic accuracy of the classical variables and the novel biomarkers (Table 3). All tested sputum biomarkers (MPO, NGAL, 
Table 2 Clinical characteristics and biomarker concentrations of the study subjects across the replication cohort $(n=135)$

\begin{tabular}{|c|c|c|c|c|c|c|}
\hline $\begin{array}{l}\text { Replication } \\
\text { cohort }\end{array}$ & $\begin{array}{l}\text { Nonsmokers } \\
(n=22)\end{array}$ & $\begin{array}{l}\text { Healthy smokers } \\
(n=40)\end{array}$ & Asthma $(n=2 I)$ & COPD $(n=35)$ & $\operatorname{Acos}(n=17)$ & $P$-value \\
\hline \multicolumn{7}{|l|}{ Demographics } \\
\hline Females/males & $13 / 9$ & $5 / 35$ & $17 / 4$ & $1 / 34$ & $3 / 14$ & \\
\hline Age (years) & $58.2(10.8)$ & $63.4(11.2)$ & $42.0(15.4)$ & $72.2(8.1)$ & $65.9(8.9)$ & $<0.001$ \\
\hline BMI $\left(\mathrm{kg} / \mathrm{m}^{2}\right)$ & $23.8(3.5)$ & $24.0(3.2)$ & $24.6(5.4)$ & $23.5(3.0)$ & $24.2(3.2)$ & 0.952 \\
\hline ICS use, $\mathrm{n}(\%)$ & - & - & $16(76)$ & - & $15(88)$ & \\
\hline Pack-years & - & 37.1 (23.7) & $4.3(12.5)$ & $47.6(21.9)$ & $40.7(16.6)$ & $<0.001$ \\
\hline \multicolumn{7}{|l|}{ Prebronchodilator } \\
\hline FVC (L) & $2.9(0.7)$ & $3.6(1.0)$ & $3.1(1.0)$ & $3.1(0.8)$ & $3.2(0.6)$ & 0.079 \\
\hline FVC\% predicted & $95.6(17.3)$ & $100.0(16.6)$ & $100.4(17.5)$ & $93.2(17.4)$ & $94.9(13.2)$ & 0.302 \\
\hline $\mathrm{FEV}_{1}(\mathrm{~L})$ & $2.4(0.6)$ & $2.8(0.8)$ & $2.5(0.7)$ & $1.9(0.5)$ & I.8 $(0.5)$ & $<0.001$ \\
\hline $\mathrm{FEV}, \%$ predicted & $96.0(16.7)$ & $94.1(17.7)$ & $94.6(16.2)$ & $69.6(16.5)$ & $64.3(15.3)$ & $<0.001$ \\
\hline $\mathrm{FEV}_{1} / \mathrm{FVC} \%$ & $83.2(7.1)$ & $77.1(5.7)$ & $80.7(6.1)$ & $59.8(8.6)$ & $55.2(11.5)$ & $<0.001$ \\
\hline \multicolumn{7}{|c|}{ Differential sputum cell counts (\%) } \\
\hline Eosinophils & $2.6(4.1)$ & $2.0(3.2)$ & $12.0(17.4)$ & $3.2(8.7)$ & $9.2(17.1)$ & 0.051 \\
\hline Neutrophils & $64.2(27.7)$ & $70.0(20.9)$ & $46.2(26.0)$ & $72.4(17.4)$ & $66.1(24.8)$ & 0.008 \\
\hline \multicolumn{7}{|c|}{ Sputum biomarker concentrations } \\
\hline IL-6 (pg/mL) & $4.4(1.5,11.4)$ & $8.3(4.0,18.2)$ & II.2(4.0, 20.1) & II.6 (3.4, 28.I) & $21.8(7.4,54.2)$ & 0.017 \\
\hline YKL-40 (ng/mL) & $10.1(4.5,27.7)$ & $18.3(5.0,29.4)$ & $17.4(8.2,21.7)$ & $21.3(11.4,31.1)$ & $20.6(13.4,30.6)$ & 0.138 \\
\hline $\mathrm{IL}-\mathrm{I} 3(\mathrm{pg} / \mathrm{mL})$ & $28.7(18.6,43.5)$ & $39.9(25.7,70.4)$ & I.7 (7.4, 47.4) & $39.6(33.1,52.0)$ & $39.1(22.4,59.2)$ & 0.032 \\
\hline NGAL (ng/mL) & $53.9(23.7,91.8)$ & $82.4(45.8,167.0)$ & $50.1(33.4,163.5)$ & $106.2(60.8,178.6)$ & $209.3(160.0,304.9)$ & $<0.001$ \\
\hline MPO (ng/mL) & $165.2(101.4,300.0)$ & $170.3(111.3,329.0)$ & $139.4(98.3,254.6)$ & $251.4(166.0,365.5)$ & I52.I (I I8.7, 307.4) & 0.330 \\
\hline
\end{tabular}

Notes: The demographics are presented as mean (SD) unless otherwise stated. The biomarker concentrations are presented as medians (IQR). P-value for the differences was analyzed by Kruskal-Wallis $H$ test. Comparisons between groups were evaluated by the Kruskal-Wallis test followed by the Mann-Whitney $U$ test.

Abbreviations: ACOS, asthma-COPD overlap syndrome; BMI, body mass index; FEV , forced expiratory volume in I second; FVC, forced vital capacity; ICS, inhaled corticosteroids; IL, interleukin; IQR, interquartile range; MPO, myeloperoxidase; NGAL, neutrophil gelatinase-associated lipocalin; SD, standard deviation; YKL-40, chitinase3-like protein I.

YKL-40, IL-6, and IL-13) could distinguish ACOS significantly from the other non-ACOS groups (AUC >0.7). Importantly, sputum biomarkers YKL-40, IL-6, and NGAL could distinguish ACOS significantly from both asthma and COPD (AUC >0.7). In addition, sputum MPO levels could differentiate ACOS from asthma, but not ACOS from COPD. Sputum IL-13 levels did not significantly separate ACOS from asthma or ACOS from COPD.

\section{Relationships between the sputum biomarkers and clinical characteristics}

The study subjects in the asthma, COPD, and ACOS groups were pooled to analyze the correlations between biomarker concentrations as the dependent factor and clinical variables. Multivariate analysis revealed pack-years and age to be the independent predictors for sputum MPO, whereas age and pre- $\mathrm{FEV}_{1} \%$ were independent predictors for YKL-40

Table 3 ROC analysis for sputum biomarkers in the discovery cohort

\begin{tabular}{|c|c|c|c|c|c|}
\hline Discovery cohort & IL-6 (pg/mL) & YKL-40 (ng/mL) & IL-I 3 (pg/mL) & NGAL $(\mu \mathrm{g} / \mathrm{mL})$ & MPO $(\mu \mathrm{g} / \mathrm{mL})$ \\
\hline \multicolumn{6}{|l|}{ ACOS versus non-ACOS } \\
\hline AUC $(95 \% \mathrm{Cl})$ & $0.887(0.8 \mid 3-0.962)$ & $0.854(0.749-0.959)$ & $0.753(0.636-0.870)$ & $0.814(0.697-0.932)$ & $0.737(0.590-0.883)$ \\
\hline Optimal threshold & $>200.5$ & $>90.4$ & $>99.7$ & $>4.0$ & $>14.5$ \\
\hline Sensitivity/specificity & $0.938 / 0.757$ & $0.833 / 0.845$ & $0.882 / 0.515$ & $0.833 / 0.750$ & $0.706 / 0.789$ \\
\hline$P$-value & $<0.001$ & $<0.001$ & 0.001 & $<0.001$ & 0.003 \\
\hline \multicolumn{6}{|l|}{ ACOS versus COPD } \\
\hline AUC $(95 \% \mathrm{Cl})$ & $0.772(0.617-0.926)$ & $0.794(0.638-0.951)$ & $0.665(0.486-0.844)$ & $0.7 I I(0.54 I-0.88 I)$ & $0.647(0.460-0.834)$ \\
\hline Optimal threshold & $>479.8$ & $>78.9$ & $>175.2$ & $>4.0$ & $>14.3$ \\
\hline Sensitivity/specificity & $0.625 / 0.850$ & $0.833 / 0.800$ & $0.647 / 0.700$ & $0.833 / 0.600$ & $0.706 / 0.650$ \\
\hline$P$-value & 0.006 & 0.002 & 0.088 & 0.026 & 0.128 \\
\hline \multicolumn{6}{|l|}{ ACOS versus asthma } \\
\hline AUC $(95 \% \mathrm{Cl})$ & $0.930(0.853-1.000)$ & $0.8 \mathrm{I} 4(0.68 \mathrm{I}-0.947)$ & $0.638(0.463-0.8 / 2)$ & $0.833(0.704-0.963)$ & $0.767(0.598-0.937)$ \\
\hline Optimal threshold & $>199.5$ & $>90.4$ & $>377.8$ & $>3.8$ & $>14.2$ \\
\hline Sensitivity/specificity & $0.938 / 0.875$ & $0.833 / 0.750$ & $0.412 / 0.864$ & $0.833 / 0.792$ & $0.706 / 0.917$ \\
\hline$P$-value & $<0.001$ & 0.001 & 0.145 & $<0.001$ & 0.004 \\
\hline
\end{tabular}

Abbreviations: ACOS, asthma-COPD overlap syndrome; AUC, area under the curve; Cl, confidence interval; IL, interleukin; MPO, myeloperoxidase; NGAL, neutrophil gelatinase-associated lipocalin; ROC, receiver operating characteristic; YKL-40, chitinase-3-like protein I. 
Table 4 Multivariate stepwise analysis of all subjects with sputum biomarker as the dependent variable in patients with asthma, COPD, and $\mathrm{ACOS}^{\mathrm{a}}$

\begin{tabular}{llll}
\hline Discovery cohort & $\boldsymbol{\beta}$ & $\boldsymbol{t}$ & $\boldsymbol{P}$-value \\
\hline Sputum IL-6 & & & \\
$\quad$ Pre-FEV \% predicted & -0.309 & $-2.38 \mathrm{I}$ & 0.021 \\
$\quad$ Pack-years & 0.262 & 2.018 & 0.049 \\
$\begin{array}{l}\text { Sputum NGAL } \\
\quad \text { Pre-FEV,\% predicted }\end{array}$ & -0.420 & 3.498 & 0.001 \\
Sputum YKL-40 & & & \\
$\quad$ Age (years) & 0.283 & 2.257 & 0.028 \\
$\quad$ Pre-FEV, \% predicted & -0.273 & 2.183 & 0.033 \\
Sputum MPO & & & \\
$\quad$ Pack-years & 0.390 & 3.304 & 0.002 \\
$\quad$ Age (years) & 0.282 & 2.388 & 0.020 \\
\hline
\end{tabular}

Notes: aMultivariate analysis was adjusted for age, body mass index, pack-years, and all the significant variables in the univariate analysis.

Abbreviations: ACOS, asthma-COPD overlap syndrome; $\mathrm{FEV}_{1}$, forced expiratory volume in I second; IL, interleukin; MPO, myeloperoxidase; NGAL, neutrophil gelatinase-associated lipocalin; YKL-40, chitinase-3-like protein I.

(Table 4). Sputum IL-6 was an independent predictor for pre- $\mathrm{FEV}_{1} \%$ predicted and pack-years, and sputum NGAL was independently associated with pre-FEV ${ }_{1} \%$ predicted (Table 4 and Figure 1A and B).

\section{Validation of the findings in an independent replication data set}

The clinical data and the levels of sputum biomarkers (MPO, NGAL, YKL-40, IL-6, and IL-13) in the replication

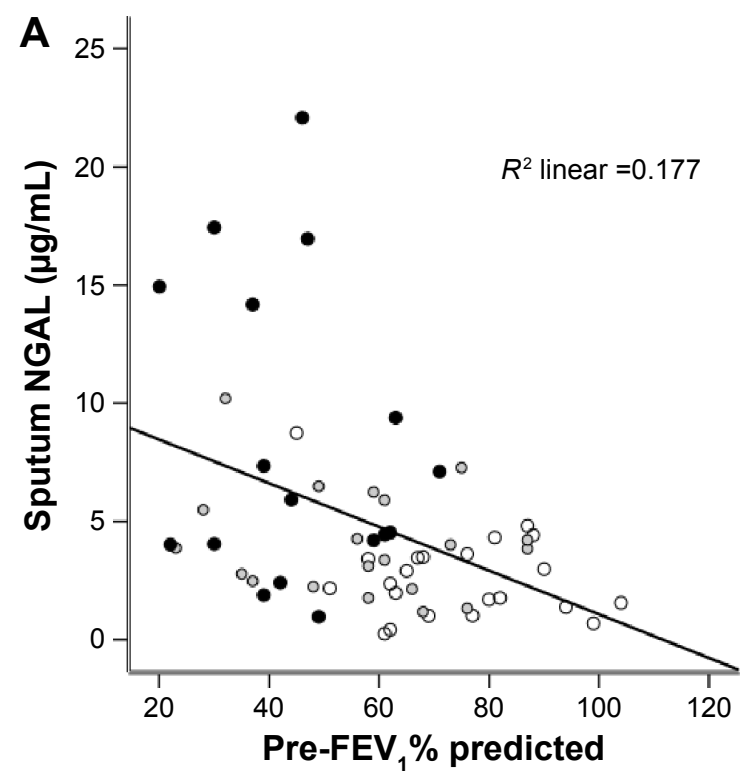

cohort are shown in Table 2. Sputum neutrophil\% count was significantly different between the groups $(P=0.008)$, and eosinophil\% count showed a tendency of differences between the groups $(P=0.051)$.

In the replication cohort, sputum IL-6, NGAL, and IL-13 levels had statistically significant differences between the study groups (Table 2). The sputum levels of IL-6 and NGAL were significantly increased in ACOS when compared with the NS ( $P=0.001$ and $P<0.001$, respectively) and the HS groups ( $P=0.018$ and $P<0.001$, respectively) (Table 2). Importantly, only sputum NGAL levels were elevated in ACOS when compared with the COPD $(P=0.002)$ and asthma $(P<0.001)$ groups (Figure 2) and had an increasing trend in COPD when compared with those in the asthma group $(P=0.068)$. Other sputum biomarkers did not differ significantly between ACOS and asthma or between ACOS and COPD in the replication cohort, but there was a significant difference in sputum IL-13 levels between asthma and COPD $(P=0.025)$.

NGAL levels could differentiate ACOS from the other study participants (non-ACOS) with an AUC value of 0.827 (95\% CI: $0.727-0.926, P<0.001)$ in the replication cohort. In addition, sputum NGAL could differentiate ACOS from the COPD and asthma groups with AUC values of 0.794 (95\% CI: 0.629-0.924, $P=0.002$ ) and 0.840 (95\% CI: 0.717-0.964, $P<0.001)$, respectively.

The asthma, COPD, and ACOS groups in the replication cohort were pooled to analyze the correlations in the univariate

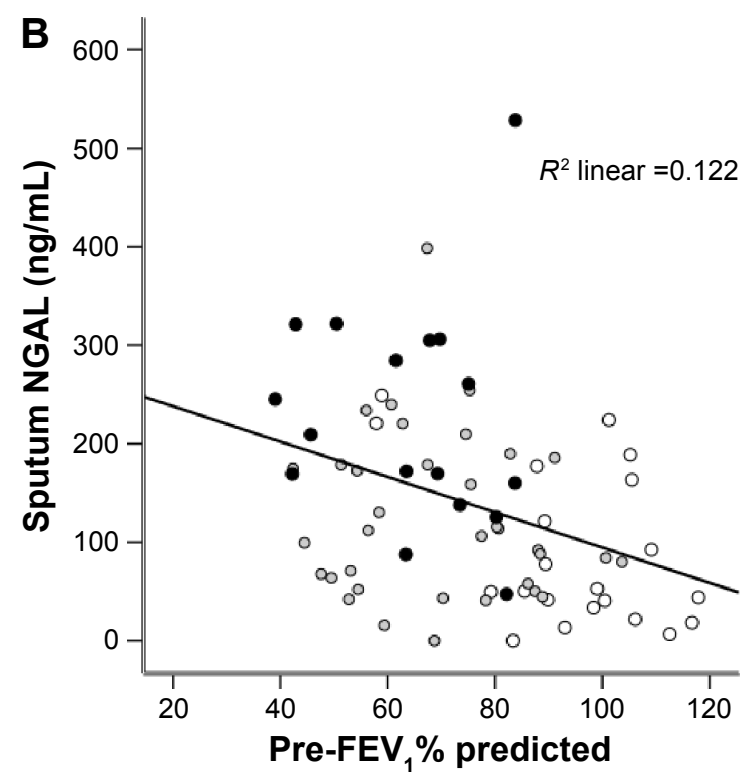

Asthma O COPD ACOS

Figure I Correlation of sputum NGAL with pre-FEV,\% predicted in patients with asthma, COPD, and ACOS in the discovery (A) and in the replication cohorts (B). Abbreviations: ACOS, asthma-COPD overlap syndrome; $\mathrm{FEV}_{1}$, forced expiratory volume in I second; NGAL, neutrophil gelatinase-associated lipocalin. 


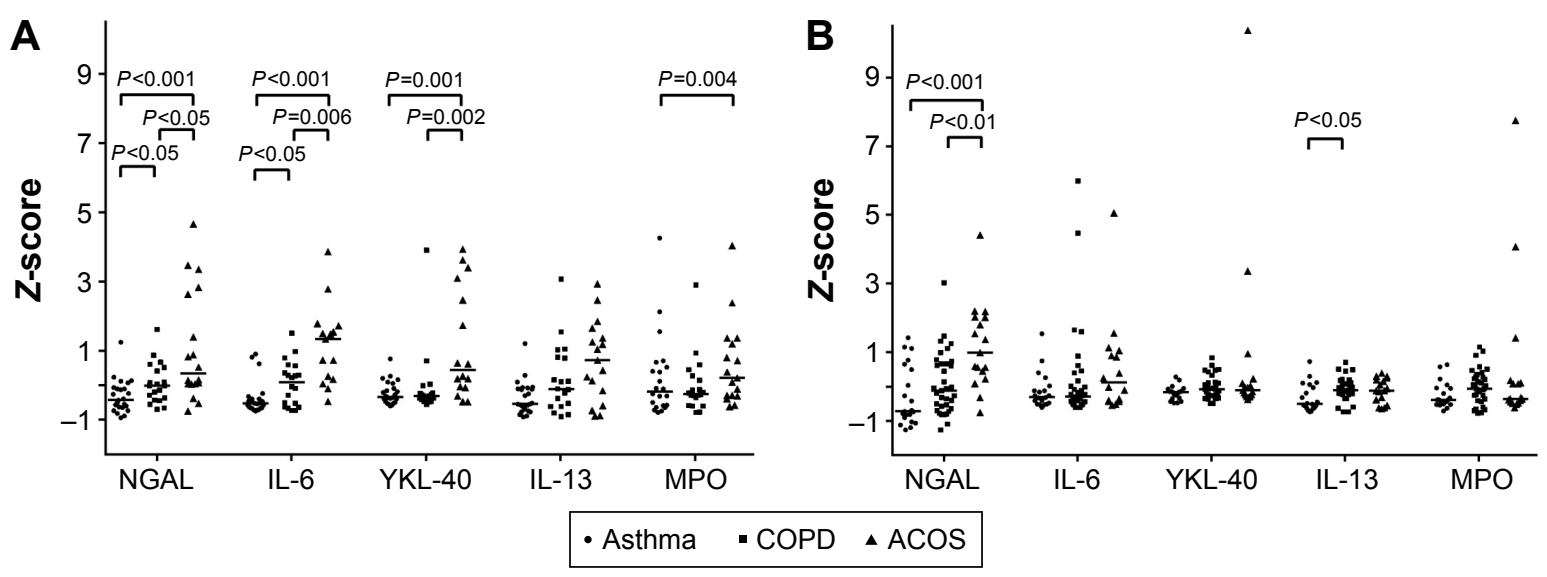

Figure 2 Expression levels of sputum biomarkers in patients with asthma, COPD, and ACOS in the discovery (A) and replication cohorts (B). Notes: The results represent standardized data (Z-scores), and the solid lines show the median value.

Abbreviations: ACOS, asthma-COPD overlap syndrome; IL, interleukin; MPO, myeloperoxidase; NGAL, neutrophil gelatinase-associated lipocalin; YKL-40, chitinase-3-like protein I.

and multivariate analyses. Sputum YKL-40 and sputum IL-6 did not correlate with any clinical parameters. Multivariate analysis revealed that sputum IL-13 correlated with packyears and sputum MPO correlated with neutrophil\% count. In agreement with the results from the discovery cohort, NGAL levels independently correlated with pre-FEV $\%$ predicted in the replication cohort $(P=0.002$, Figure 1B).

\section{Discussion}

This study analyzed the biomarkers related to asthma or COPD or implicated in both diseases to discover inflammatory profiles typical for ACOS in two independent cohorts. The levels of sputum IL-13 and MPO were higher in the ACOS patients than in the healthy controls in the discovery cohort, but this was not apparent in the replication cohort. In both cohorts, the sputum levels of NGAL, IL-6, and YKL-40 were elevated in ACOS when compared with those of NSs. However, only sputum NGAL could differentiate ACOS from COPD and asthma in both cohorts.

The most recent treatment guidelines present features that characterize ACOS rather than provide a formal definition of ACOS. ${ }^{33}$ Four distinct pathways have been proposed for ACOS. ${ }^{34}$ Airflow limitation in early life can persist through adolescence into adulthood and ACOS is more likely than severe asthma if other risk factors, such as smoking, are present. A second pathway is represented in COPD patients with substantial smoking histories or other exposures and late-onset features of asthma. A third asthma-dominant pathway is represented by asymptomatic adults with airway hyperresponsiveness who progress to chronic airflow limitation compatible with a diagnosis of COPD. Finally, a fourth pathway recognizes the link between early-life risk factors and small lungs, with an increased risk of development of fixed airflow limitation and asthma. Thus, from the molecular genetic point of view, ACOS can represent a very heterogeneous group of patients. All patients with ACOS in the discovery cohort were either current or former smokers. There were eleven patients who mostly represent the second COPD-dominant pathway in the discovery cohort, and seven patients who developed fixed airway limitation after asthma diagnosis. There was more variation in the biomarker levels in the replication cohort than in the discovery cohort suggesting that there may be more etiological variability between the patients. The final goal would be to identify a panel of markers with the optimal capability to dissect the endotypes valid for prognosis and in treatment choices.

Elevated IL-6 and YKL-40 levels were observed in patients with ACOS in both cohorts although the differences between patients with ACOS, asthma, and COPD were not statistically significant in the replication cohort. This could be explained by the higher etiological variability between the patients or by the milder airway limitation. In a recent study, elevated serum IL-6 levels were detected in patients with ACOS, although systemic IL-6 levels did not differentiate COPD from ACOS. ${ }^{22}$ Circulating biomarkers may also not be specific for airway disease unlike sputum samples and there is no direct link between systemic and airway inflammatory mediators. ${ }^{35}$

The important finding of this study was that sputum NGAL levels were significantly increased in patients with ACOS in comparison with the levels in the asthma and COPD groups in two independent cohorts. Therefore, although ACOS might be a complex and heterogeneous disease, the results of the current study further support that increased sputum 
NGAL might be a characteristic feature of ACOS. Although MPO and NGAL are both COPD-related biomarkers, MPO is associated with a local activation of neutrophils, $, 936,37$ whereas NGAL is not only attributed to activated neutrophils but could also be secreted by the respiratory epithelial cells in response to inflammatory stimuli ${ }^{9,38}$ and by myeloid and epithelial cells in response to toll-like receptor activation during bacterial infections. ${ }^{39,40}$ Patients with ACOS seem to have more frequent exacerbations, ${ }^{41,42}$ and microorganisms are one of the main etiologic factor involved in exacerbations of COPD. Therefore, the sputum NGAL levels might be related to airway inflammation and low-grade microbial colonization, which predispose patients with ACOS to acute viral infections and exacerbations.

In addition, earlier reports have shown that NGAL expression in lung can be induced by reactive oxygen species. ${ }^{43,44}$ Because the pathophysiology of both asthma and COPD is associated with oxidative processes induced by environmental exposures and airway inflammation, ${ }^{45}$ ACOS might have augmented oxidative stress that could result in airway reactivity, injury, and remodeling and might also relate to increased sputum NGAL levels. Finally, it is important that increased sputum NGAL was independently correlated with degree of airflow limitation in both the discovery and replication cohorts. This finding indicates that sputum NGAL may be a candidate marker for airway remodeling, but the clinical utility of this finding should be tested in a future prospective study.

Although many biomarkers examined in this study are likely to be associated with other morbidities, the use of sputum may improve their specificity for airway disease. The changes in sputum biomarker levels are likely to reflect the changes of cell composition and lung function during the development of chronic airway disease. Therefore, this study results suggest that ACOS could be characterized by mediators related to airway inflammation and airway remodeling. Future studies with high-throughput design are required to fully reveal the transcriptomic or proteomic differences in chronic airway disease.

The strength of this study design was that the control subjects did not have any other exposures, and the diagnosis criteria for asthma, COPD, and ACOS were based on international guidelines, sputum cell counts, and clinical characteristics of the disease. The sample size was limited, but the obtained results were replicated in another independent cohort.

\section{Conclusion}

The results from the current study indicate that ACOS is associated with sputum biomarkers that drive both airway inflammation and tissue remodeling, and these biomarkers could have diagnostic value in differentiation of ACOS from asthma and COPD.

\section{Acknowledgments}

The authors would like to thank Professor Vuokko Kinnula for her contribution prior to her death on November 17 , 2012. Ms Tinja Kanerva, Tiina Lapinkari, Kerstin Alhskog, Kirsi Elorinne, Sari Tillander, Yukari Iyanaga, Kakuhiro Yamaguchi, Naoko Higaki, and Shuai Ni are acknowledged for their help and excellent technical assistance. The authors also thank Doctor Päivi Piirilä for her help at accessing lung function tests. This work was financially supported by the EVO funding of the Helsinki University Central Hospital, Research Funds of the University of Helsinki, Sigrid Jusélius Foundation, Finnish Anti-Tuberculosis Association Foundation, the Jalmari and Rauha Ahokas Foundation, the Finnish Cultural Foundation, and the SalWe Research Program for IMO (Tekes - the Finnish Funding Agency for Technology and Innovation grant 648/10). Jing Gao was further supported by the China Scholarship Council, CIMO, the Research Foundation of the Pulmonary Diseases, Ida Montin Foundation, and Väinö and Laina Kivi Foundation.

\section{Disclosure}

The authors report no conflicts of interest in this work.

\section{References}

1. Vestbo J, Hurd SS, Agusti AG, et al. Global strategy for the diagnosis, management, and prevention of chronic obstructive pulmonary disease: GOLD executive summary. Am J Respir Crit Care Med. 2013;187(4): 347-365.

2. Reddel HK, Bateman ED, Becker A, et al. A summary of the new GINA strategy: a roadmap to asthma control. Eur Respir J. 2015;46(3): 622-639.

3. Postma DS, Rabe KF. The asthma-COPD overlap syndrome. $N$ Engl $J$ Med. 2015;373(13):1241-1249.

4. Gibson PG, McDonald VM. Asthma-COPD overlap 2015: now we are six. Thorax. 2015;70(7):683-691.

5. Gibson PG, Simpson JL. The overlap syndrome of asthma and COPD: what are its features and how important is it? Thorax. 2009;64(8): 728-735.

6. Kauppi P, Kupiainen H, Lindqvist A, et al. Overlap syndrome of asthma and COPD predicts low quality of life. J Asthma. 2011;48(3): 279-285.

7. Marsh SE, Travers J, Weatherall M, et al. Proportional classifications of COPD phenotypes. Thorax. 2008;63(9):761-767.

8. Andersen H, Lampela P, Nevanlinna A, Saynajakangas O, Keistinen T. High hospital burden in overlap syndrome of asthma and COPD. Clin Respir J. 2013;7(4):342-346.

9. Iwamoto H, Gao J, Koskela J, et al. Differences in plasma and sputum biomarkers between COPD and COPD-asthma overlap. Eur Respir J. 2014;43(2):421-429.

10. Hardin M, Silverman EK, Barr RG, et al; COPDGene Investigators. The clinical features of the overlap between COPD and asthma. Respir Res. 2011;12:127. 
11. Barnes PJ. The cytokine network in asthma and chronic obstructive pulmonary disease. J Clin Invest. 2008;118(11):3546-3556.

12. Barnes PJ. Immunology of asthma and chronic obstructive pulmonary disease. Nat Rev Immunol. 2008;8(3):183-192.

13. Barrecheguren M, Esquinas C, Miravitlles M. The asthma - chronic obstructive pulmonary disease overlap syndrome (ACOS): opportunities and challenges. Curr Opin Pulm Med. 2015;21(1):74-79.

14. Tamada T, Sugiura H, Takahashi T, et al. Biomarker-based detection of asthma-COPD overlap syndrome in COPD populations. Int J Chron Obstruct Pulmon Dis. 2015;10:2169-2176.

15. Dente FL, Bacci E, Vagaggini B, Paggiaro P. Cytokines in induced sputum: a role for the ratio of IL-6/IL-13 in the differentiation of asthma and chronic obstructive pulmonary disease? Respiration. 2012; 84(2):98-100.

16. Wills-Karp M, Luyimbazi J, Xu X, et al. Interleukin-13: central mediator of allergic asthma. Science. 1998;282(5397):2258-2261.

17. Tang H, Fang Z, Sun Y, et al. YKL-40 in asthmatic patients, and its correlations with exacerbation, eosinophils and immunoglobulin E. Eur Respir J. 2010;35(4):757-760.

18. Otsuka K, Matsumoto H, Niimi A, et al. Sputum YKL-40 levels and pathophysiology of asthma and chronic obstructive pulmonary disease. Respiration. 2012;83(6):507-519.

19. James AJ, Reinius LE, Verhoek M, et al. Increased YKL-40 and chitotriosidase in asthma and chronic obstructive pulmonary disease. Am J Respir Crit Care Med. 2016;193(2):131-142.

20. Grubek-Jaworska H, Paplinska M, Hermanowicz-Salamon J, et al. IL-6 and IL-13 in induced sputum of COPD and asthma patients: correlation with respiratory tests. Respiration. 2012;84(2):101-107.

21. Attaran D, Lari SM, Towhidi M, et al. Interleukin-6 and airflow limitation in chemical warfare patients with chronic obstructive pulmonary disease. Int J Chron Obstruct Pulmon Dis. 2010;5:335-340.

22. Fu JJ, McDonald VM, Gibson PG, Simpson JL. Systemic inflammation in older adults with asthma-COPD overlap syndrome. Allergy Asthma Immunol Res. 2014;6(4):316-324.

23. Laitinen T, Hodgson U, Kupiainen H, et al. Real-world clinical data identifies gender-related profiles in chronic obstructive pulmonary disease. COPD J Chronic Obstr Pulmonary Dis. 2009;6(4):256-262.

24. British Thoracic Society Scottish Intercollegiate Guidelines N. British guideline on the management of asthma. Thorax. 2008;63 Suppl 4: iv1-iv121.

25. Miller MR, Hankinson J, Brusasco V, et al. Standardisation of spirometry. Eur Respir J. 2005;26(2):319-338.

26. Enright P, Skloot G, Herbert R. Standardization of spirometry in assessment of responders following man-made disasters: World Trade Center worker and volunteer medical screening program. Mt Sinai J Med. 2008;75(2):109-114.

27. Ishikawa N, Hattori N, Kohno N, Kobayashi A, Hayamizu T, Johnson M. Airway inflammation in Japanese COPD patients compared with smoking and nonsmoking controls. Int J Chron Obstruct Pulmon Dis. 2015;10:185-192.

28. Viljanen AA, Halttunen PK, Kreus KE, Viljanen BC. Spirometric studies in non-smoking, healthy adults. Scand J Clin Lab Invest Suppl. 1982;159:5-20.
29. Djukanovic R, Sterk PJ, Fahy JV, Hargreave FE. Standardised methodology of sputum induction and processing. Eur Respir J Suppl. 2002; $37: 1 \mathrm{~s}-2 \mathrm{~s}$.

30. Kelly MM, Keatings V, Leigh R, et al. Analysis of fluid-phase mediators. Eur Respir J Suppl. 2002;37:24s-39s.

31. Louhelainen N, Stark H, Mazur W, Rytila P, Djukanovic R, Kinnula VL. Elevation of sputum matrix metalloproteinase-9 persists up to 6 months after smoking cessation: a research study. BMC Pulm Med. 2010;10:13.

32. Shiota N, Yokoyama A, Haruta Y, Hattori N, Kohno N. Association of airway inflammation with asthma control level evaluated by the asthma control test. J Asthma. 2011;48(9):907-913.

33. Boulet LP, FitzGerald JM, Reddel HK. The revised 2014 GINA strategy report: opportunities for change. Curr Opin Pulm Med. 2015; 21(1):1-7.

34. Bateman ED, Reddel HK, van Zyl-Smit RN, Agusti A. The asthmaCOPD overlap syndrome: towards a revised taxonomy of chronic airways diseases? Lancet Respir Med. 2015;3(9):719-728.

35. Donaldson GC, Seemungal TAR, Patel IS, et al. Airway and systemic inflammation and decline in lung function in patients with COPD. Chest. 2005;128(4):1995-2004.

36. Keatings VM, Barnes PJ. Granulocyte activation markers in induced sputum: comparison between chronic obstructive pulmonary disease, asthma, and normal subjects. Am J Respir Crit Care Med. 1997;155(2) 449-453.

37. Aaron SD, Angel JB, Lunau M, et al. Granulocyte inflammatory markers and airway infection during acute exacerbation of chronic obstructive pulmonary disease. Am J Respir Crit Care Med. 2001; 163(2):349-355.

38. Cowland JB, Sorensen OE, Sehested M, Borregaard N. Neutrophil gelatinase-associated lipocalin is up-regulated in human epithelial cells by IL-1 beta, but not by TNF-alpha. J Immunol. 2003;171(12): 6630-6639.

39. Flo TH, Smith KD, Sato S, et al. Lipocalin 2 mediates an innate immune response to bacterial infection by sequestrating iron. Nature. 2004; 432(7019):917-921.

40. Chan YR, Liu JS, Pociask DA, et al. Lipocalin 2 is required for pulmonary host defense against Klebsiella infection. J Immunol. 2009;182(8): 4947-4956.

41. Nielsen M, Barnes CB, Ulrik CS. Clinical characteristics of the asthmaCOPD overlap syndrome - a systematic review. Int J Chron Obstruct Pulmon Dis. 2015;10:1443-1454.

42. Menezes AM, Montes de Oca M, Perez-Padilla R, et al. Increased risk of exacerbation and hospitalization in subjects with an overlap phenotype: COPD-asthma. Chest. 2014;145(2):297-304.

43. Roudkenar MH, Kuwahara Y, Baba T, et al. Oxidative stress induced lipocalin 2 gene expression: addressing its expression under the harmful conditions. J Radiat Res. 2007;48(1):39-44.

44. Sunil VR, Patel KJ, Mainelis G, et al. Pulmonary effects of inhaled diesel exhaust in aged mice. Toxicol Appl Pharmacol. 2009;241(3): 283-293.

45. Erzurum SC. New insights in oxidant biology in asthma. Ann Am Thorac Soc. 2016;13(suppl 1):S35-S39.
International Journal of COPD

\section{Publish your work in this journal}

The International Journal of COPD is an international, peer-reviewed journal of therapeutics and pharmacology focusing on concise rapid reporting of clinical studies and reviews in COPD. Special focus is given to the pathophysiological processes underlying the disease, intervention programs, patient focused education, and self management protocols.

\section{Dovepress}

This journal is indexed on PubMed Central, MedLine and CAS. The manuscript management system is completely online and includes a very quick and fair peer-review system, which is all easy to use. Visit http://www.dovepress.com/testimonials.php to read real quotes from published authors. 